\title{
Ferromagnet-free all-electric spin Hall transistors
}

Won Young Choi , Hyung-jun Kim, Joonyeon Chang, Suk Hee Han', Adel Abbout, Hamed Ben Mohamed Saidaoui ${ }^{\ddagger}$, Aurélien Manchon $*$, Kyung-Jin Lee ${ }^{\|, \perp}$, and Hyun Cheol Koo**..

Center for Spintronics, Korea Institute of Science and Technology, Seoul, 02792, Korea

King Abdullah University of Science and Technology (KAUST), Physical Science and Engineering Division (PSE), Thuwal 23955-6900, Saudi Arabia

King Abdullah University of Science and Technology (KAUST), Computer, Electrical and Mathematical Science and Engineering Division (CEMSE), Thuwal 23955-6900, Saudi Arabia

"KU-KIST Graduate School of Converging Science and Technology, Korea University, Seoul

02841, Korea

${ }^{\perp}$ Department of Materials Science and Engineering, Korea University, Seoul 02841, Korea

KEYWORDS: Spin transistor, spin logic device, spin Hall effect, Rashba effect, spin precession 
ABSTRACT: Spin field effect transistor, an essential building block for spin information processing, shows promise for energy-efficient computing. Despite steady progress, it suffers from a low output signal because of low spin injection and detection efficiencies. We demonstrate that this low-output obstacle can be overcome by utilizing direct and inverse spin Hall effects for spin injection and detection, respectively, without a ferromagnetic component. The output voltage of our all-electric spin Hall transistor is about two orders of magnitude larger than previously reported spin transistors based on ferromagnets or quantum point-contacts. Moreover, the symmetry of spin Hall effect allows all-electric spin Hall transistors to effectively mimic $n$-type and $p$-type devices, opening a way of realizing the complementary functionality.

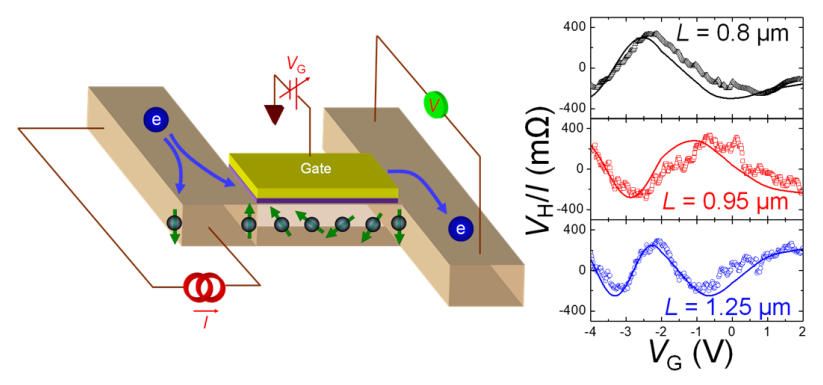


The manipulation of electron spins by the spin-orbit interaction lies at the heart of condensed matter physics. A representative example is the spin precession around an effective spin-orbit field, which serves as a key operation principle of spin field effect transistor (spin-FET) through the gate-controlled spin-orbit interaction and subsequent periodic modulation of source-drain conductance $^{1-6}$. Another intriguing ability of the spin-orbit interaction is the conversion from a charge current to a spin current, and vice versa, named direct and inverse spin Hall effects, respectively ${ }^{7-13}$. In contrast to the magnetic exchange interaction for which conversion efficiency from a charge current to a spin current (i.e., spin polarization) is limited to $100 \%$, the spin-orbit interaction allows for much larger conversion efficiency ${ }^{14,15}$, enabling energy-efficient spintronic devices.

In this work, we experiment on an all-electric spin Hall transistor that utilizes the full potential of the spin-orbit interaction by exploiting not only the spin-orbit-induced spin precession but also the direct and inverse spin Hall effects as the spin injection and detection schemes, respectively. Up to now, the spin injection and detection have been implemented by using ferromagnets ${ }^{3}$ or quantum point-contacts ${ }^{16}$. The spin injection/detection efficiency through the interface between a metallic ferromagnet and a semiconducting channel is known to be extremely low because of the conductivity mismatch ${ }^{17}$. While tunneling barrier improves injection efficiency ${ }^{18}$, the increase of interfacial resistance will degrade the device performance. Recently, electric field modulation of spin current has been reported in a two-dimensional material ${ }^{19}$, but it also requires spin injection from the ferromagnetic electrode. The quantum point-contact scheme has realized the gate control of spin current but this design is fundamentally limited as the spin precession signal survives only for a point-contact conductance of around $2 e^{2} / h$, where $e$ is the electron charge and $h$ is the Planck constant $^{16}$. Moreover, its scheme requires an additional current at the detection part to get an output 
signal, resulting in unwanted complexity of the device structure. On the other hand, there are several works that utilize the inverse spin Hall effect for the spin detection. However, they are combined with optic ${ }^{112022}$ or magnetic ${ }^{2223}$ elements for the spin injection and thus the all-electric spin Hall transistor, which is of technological relevance, has not been demonstrated yet. As no interface is involved in the spin injection and detection processes, the all-electric spin Hall transistor is expected to yield a largely improved output signal as compared to other existing spin-FETs.

To implement an all-electric spin Hall transistor, we fabricated H-bar devices using an InAsbased quantum well channel that has a strong spin-orbit interaction. The operation mechanisms of this device are the spin Hall effect and the gate-controlled spin precession, all arising from the same Rashba spin-orbit coupling as illustrated in Figure 1a. The device consists of three regions. The left part of the device (region I) is the injection region where the bias current is applied in the $y$-direction. The direct spin Hall effect converts this charge current to a spin current. Because of the symmetry of spin Hall effect,

$$
\mathbf{I}_{\mathbf{s}} \propto \boldsymbol{\sigma} \times \mathbf{I}_{\mathrm{c}},
$$

where $\mathbf{I}_{s}\left(\mathbf{I}_{c}\right)$ is the flow direction of spin (charge) current and $\boldsymbol{\sigma}$ is the spin polarization direction carried by the spin current, the spins polarized along the $+z$-direction move to the $+x$-direction while the spins polarized along the $-z$-direction move to the $-x$-direction. Thus a pure spin current polarized in the $+z$-direction is injected to the middle part of the device (region II) where the gate electrode is deposited on top of it. As there is no interface between these two regions, the spin current is efficiently injected into the gate-controlled region without much loss. In the region II, the spin precession is modulated by gating. The gate voltage $V_{\mathrm{c}}$ controls the Rashba spin-orbit interaction and subsequently modulates the spin precession rate. The gate-modulated spin current 
is then injected to the right part of the device (region III), where the inverse spin Hall effect converts the spin current to a charge current, thereby generating a Hall voltage along the $y$ direction. No interface between the regions II and III also guarantees an efficient detection of spin information. As both inverse and direct spin Hall effects share the same symmetry in eq 1 through the Onsager reciprocity ${ }^{24}$, the Hall voltage oscillates with the $z$-component of injected spin, which is controlled by the gate voltage. This three-step process provides the essential functionality of allelectric spin Hall transistor without involving a ferromagnetic electrode or an external magnetic field.

The top view of the device structure is shown in Figure 1b. The device consists of an InAs high electron mobility transistor (HEMT) channel and a gate electrode (see Supplementary Information Section 1). The InAs-based quantum well was grown by molecular beam epitaxy on a semi-insulating InP (100) substrate. The asymmetric quantum well induces a strong Rashba spinorbit interaction even without a gate electric field. The carrier density and mobility of the HEMT channel are respectively $n_{\mathrm{s}}=2 \times 10^{12} \mathrm{~cm}^{-2}$ and $\mu=60,000 \mathrm{~cm}^{2} \mathrm{~V}^{-1} \mathrm{~s}^{-1}$ at $1.8 \mathrm{~K}$. The representative channel formed by ion milling had a width of $W=0.7 \mu \mathrm{m}$ and a probe width of $W_{\text {н }}=0.2 \mu \mathrm{m}$.

We first measure the Rashba parameter $\alpha$ of the channel as a function of the gate voltage $V_{\mathrm{c}}$ (Figure 1c), using the Shubnikov-de Haas oscillations ${ }^{32526}$. The gate voltage controllability is more effective for the negative $V_{\mathrm{G}}$ due to the non-linear band bending of the quantum well. Figure 1d shows a representative result of the Hall voltage detected at the region III as a function of the gate voltage for a bias current of $0.1 \mathrm{~mA}$ in the region I. It clearly shows an oscillatory Hall voltage modulation by gating. We repeat the same measurement with a reversed bias current. Because of the symmetry of spin Hall effect in eq 1, the spin orientation of spin current injected into the region 
II is reversed when the bias current is reversed. At a given gate voltage, the spins polarized in the $+z$ and $-z$ directions undergo the same amount of precession inside the region II, so that the Hall voltages for positive and negative bias currents always retain a phase difference of $180^{\circ}$ for the entire range of the gate voltages, which is consistent with what we measure.

From the Datta-Das transistor model with including exponential decay governed by the mean free path $(l)$, the detected Hall voltage can be expressed as ${ }^{123}$

$$
V_{H}=A \cos \left(2 m^{*} \alpha L / \hbar^{2}\right) e^{-L / l},
$$

where $A$ is a constant, $L$ is the channel length of region II, $\hbar$ is the reduced Planck constant, and $m^{*}=0.05 m_{0}$ is the effective mass of electron ${ }^{327}$. We find that the oscillatory behaviour of measured signals is described reasonably well by solid fitting lines, obtained from the above equation where $\alpha$ is the measured $V_{\mathrm{G}}$-dependent Rashba parameter (Figure 1c). We also measure the channel length dependence of signals by varying the distance $L$ from $0.55 \mu \mathrm{m}$ to $1.5 \mu \mathrm{m}$. From the Datta and Das model as described in eq 2, the total spin precession inside the channel is proportional to the channel length $L$. As a result, the oscillation period of Hall voltage decreases with increasing $L$ as shown in Figure 2. Based on the experimental data taken for $-4 \mathrm{~V} \leq V_{\mathrm{G}} \leq+2 \mathrm{~V}$, the half periods of oscillations are $\Delta V_{\mathrm{G}}=\sim 3 \mathrm{~V}(L=0.8 \mu \mathrm{m}), \Delta V_{\mathrm{c}}=\sim 2 \mathrm{~V}(L=0.95 \mu \mathrm{m})$, and $\Delta V_{\mathrm{G}}=\sim 1.3 \mathrm{~V}(L=1.25$ $\mu \mathrm{m})$, respectively. The solid fitting lines with eq 2 describe the experimental results reasonably in both oscillatory behavior and channel length dependence. To better understand these experimental results, we also perform numerical simulations based on a tight-binding model (see Supplementary Information Section 2). These simulations account for a large number of modes present in the device and reproduce semi-quantitatively the experimental results. We emphasize that decoherence induced by destructive interferences between the multiple modes enhanced by 
momentum scattering is crucial to obtain the experimental variation of the Hall voltage as a function of the channel length. All the experimental and numerical results explain above evidence that the three-step process, the spin injection by the direct spin Hall effect, the spin precession by the gate-controlled Rashba parameter, and the spin detection by the inverse spin Hall effect, is realized in our device.

We next discuss the magnitude of the output signal, which is the main focus of this work. In spin-FETs, an important parameter for the output signal is the width $W_{\mathrm{н}}$ of the detection part. Figure 3a shows that the output signal for $W_{\text {н }}=0.2 \mu \mathrm{m}$ is 20 times larger than that for $W_{\mathrm{н}}=2 \mu \mathrm{m}$. This strong dependence of output signal on $W_{\text {н }}$ arises because the measured Hall voltage is an averaged value of oscillatory signal over $W_{\text {н }}$ as shown in the schematic diagram of Figure $3 a$ (see Supplementary Information section 3). For a legitimate comparison of output signals among different types of spin-FETs, therefore, one has to fabricate nominally identical device structure including the width $W_{\text {н. }}$ We do so for three types of spin transistor designs exploiting the gatecontrolled Rashba effect. They are the conventional Datta-Das spin-FET using ferromagnets $\left(\mathrm{Ni}_{81} \mathrm{Fe}_{19}\right.$ electrodes) for both spin injection and detection ${ }^{3}$, the spin Hall transistor using a ferromagnet (inverse spin Hall effect) for spin injection (detection) ${ }^{23}$, and the ferromagnet-free allelectric spin Hall transistor (this work) as shown in Figure 3b. We find that the signal of the allelectric spin Hall transistor $(\sim 900 \mathrm{~m} \Omega)$ is much larger than those of two other transistors $(<40$ $\mathrm{m} \Omega)^{3,23}$. As the signals of these spin transistors are proportional to $\eta^{2}, \eta \theta_{\mathrm{eff}}$, and $\theta_{\mathrm{eff}}{ }^{2}$, respectively, where $\eta$ is the spin injection efficiency at the interface of ferromagnet/quantum well channel and $\theta_{\text {eff }}$ is the effective spin Hall angle of the channel. From the experimental results, $\theta_{\text {eff }}$ is estimated to be 0.103 . We note that previous studies with topological insulators ${ }^{14,15}$ reporting large effective spin Hall angles suggest that this ratio can become even larger. This result shows that ferromagnet-free 
all-electric spin Hall transistors are superior to other types of spin transistors and the spin Hall effect provides a much more efficient injection/detection efficiency than that with ferromagnets.

We finally demonstrate $p$ - and $n$-type all-electric spin Hall transistors, which are essential for the complementary functionality in logic applications. The symmetry of spin Hall effect in eq 1 allows for an easy implementation of $p$ - and $n$-type transistors simply by relocating output terminals without an additional doping process. As shown in Figure 4a, the $U$-type ( $D$-type) spin transistor has one of the output terminals at upper (lower) side of the detection part while the other terminal is located at the right end of the horizontal channel. The inverse spin Hall effect generates charge accumulations with the opposite sign at the upper and lower sides of the detection part. As shown in Figure 4b, the two types of transistors always produce complementary signals for all gate voltages. Figure $4 \mathrm{c}$ shows that for $V_{\mathrm{c}}=+1.4 \mathrm{~V}$, the $U$-type transistor reads high potential whereas the $D$-type transistor reads low potential at the output terminals. On the other hand, for $V_{\mathrm{G}}=-1.4$ $\mathrm{V}$, the $U$-type ( $D$-type) transistor produces low (high) potential. Thus, the $U$ - and the $D$-type spin Hall transistors are able to respectively mimic the conventional $p$ - and $n$-type transistors for logic applications.

The spin Hall and Rashba effects have fostered great interest because they connect the electron charge and spin even without a ferromagnetic material. Our proposed ferromagnet-free all-electric spin Hall transistor provides an alternative operation scheme with much improved signals than previously realized spin-based transistors, as it avoids the hurdles inherent to spin injection and detection at ferromagnet-semiconductor interfaces. The next step of spin transistor research will be the realization of room-temperature operation, which requires the development of a channel material with a spin-orbit splitting energy much larger than the thermal energy. In this respect, recent experiment ${ }^{28}$ and theory ${ }^{29}$, which reported an unprecedented spin splitting of 150 
meV and emphasized the important role of the inversion symmetry breaking, would shed light on this direction. 
a
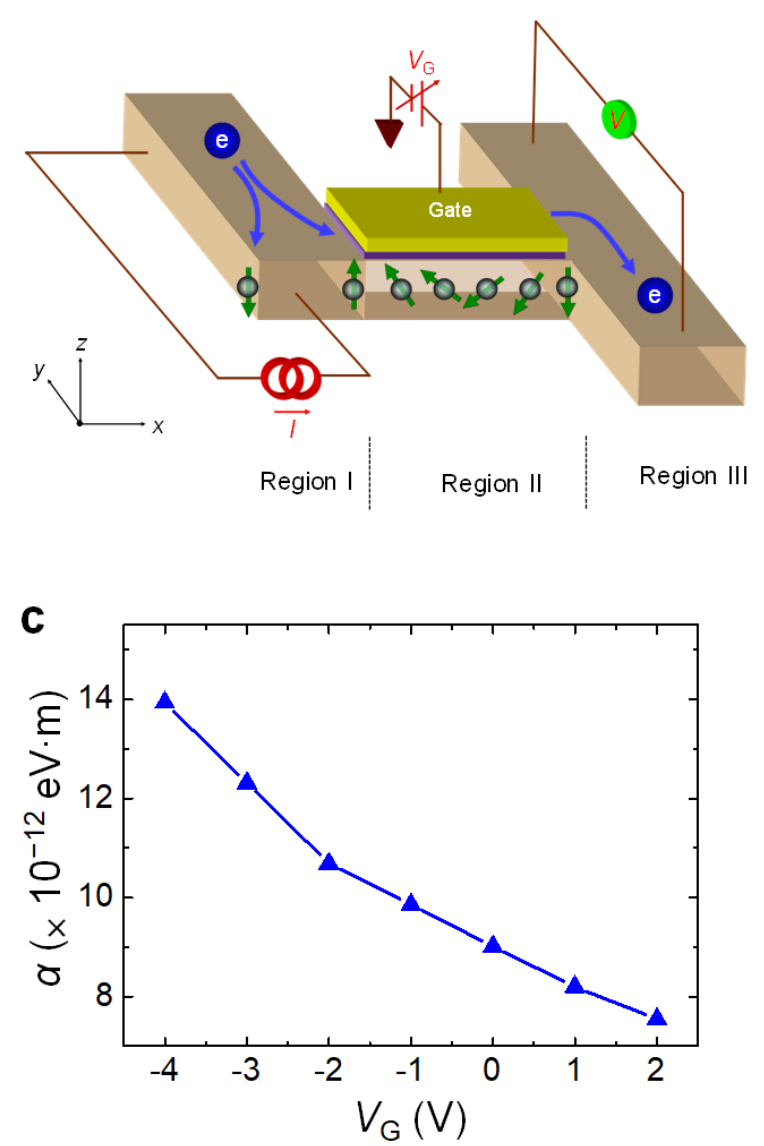

b
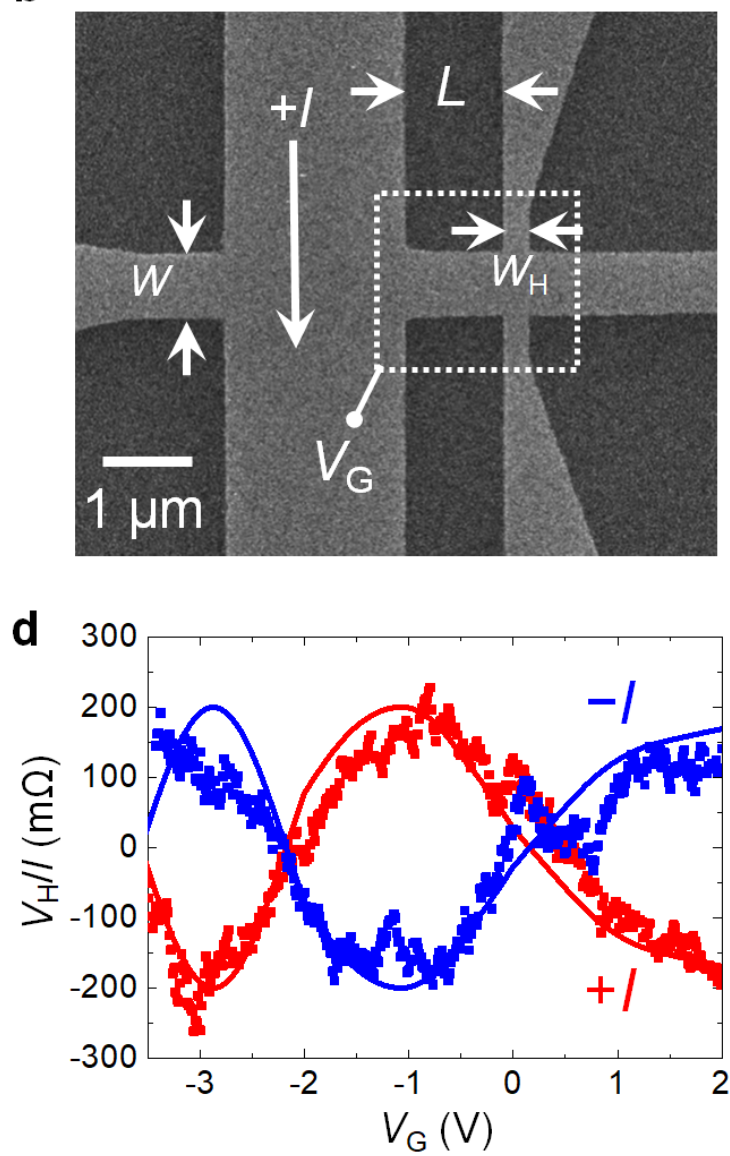

Figure 1. Ferromagnet-free spin transistor. (a) Schematic diagram of device. (b) Scanning electron micrograph of device before forming gate electrode. The image was taken before gate formation for clarity. (c) Gate-controlled Rashba parameter. (d) Gate control of Hall voltage. Red trace: bias current I positive. Blue trace: I negative. The channel length is $0.95 \mu \mathrm{m}$, bias current is $0.1 \mathrm{~mA}$, and the measuring temperature is $1.8 \mathrm{~K}$. The offset resistance is $650 \mathrm{~m} \Omega$. The solid line fits in $\mathrm{d}$ are from the Datta-Das model and the gate voltage dependence of Rashba parameter shown in (c). 


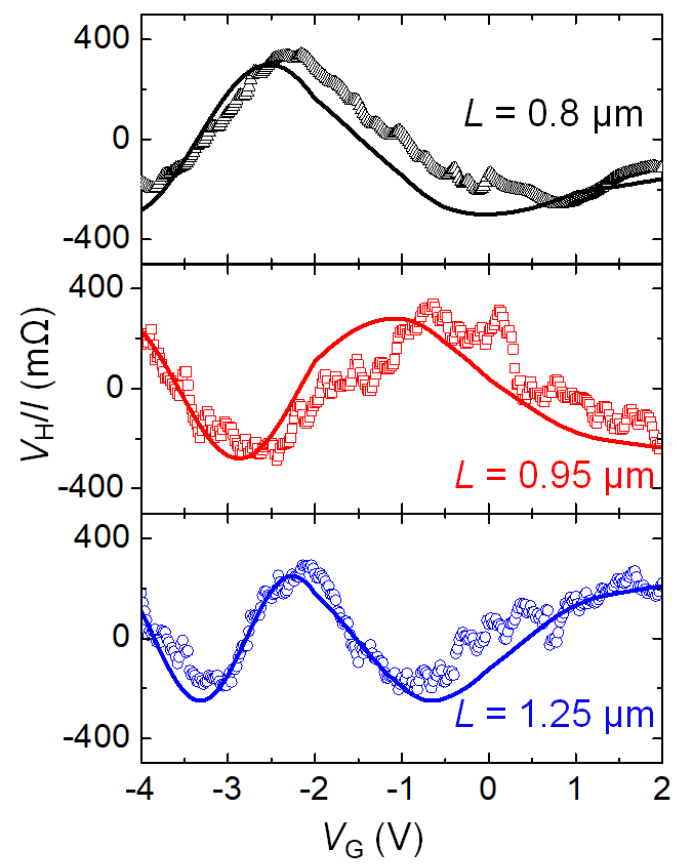

Figure 2. Gate-controlled spin signal for $W_{\text {н }}=0.2 \mu \mathrm{m}$. The bias current is $0.1 \mathrm{~mA}$, and the measuring temperature is $1.8 \mathrm{~K}$. Offset resistances are $1.8 \Omega, 0.4 \mathrm{~m} \Omega$, and $\sim 0 \Omega$ for $L=0.8 \mu \mathrm{m}$, $0.95 \mu \mathrm{m}$, and $1.25 \mu \mathrm{m}$, respectively. The solid line fits are from the experimentally determined Rashba parameters, $\alpha\left(V_{\mathrm{c}}\right)$ and the independently measured mean free path, $l=1.61 \mu \mathrm{m}$. 

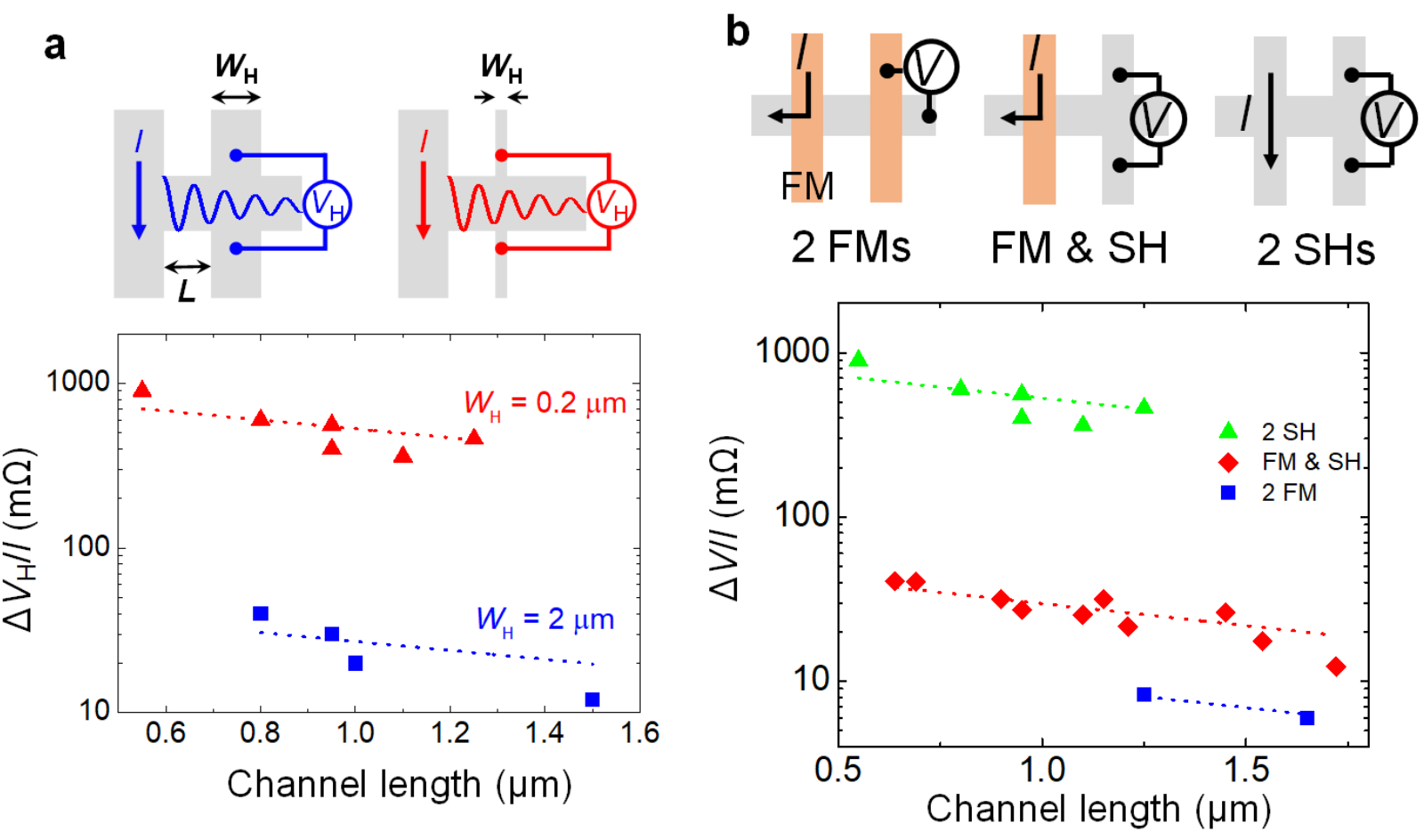

Figure 3. Magnitude of spin transistor signal. (a) Channel length dependence of magnitude for the Hall probe widths of $0.2 \mu \mathrm{m}$ and $2 \mu \mathrm{m}$. The dotted line fits are from the independently measured mean free path, $l=1.61 \mu \mathrm{m}$. The upper diagrams show that the larger $W_{\mathrm{н}}$ reflects the wider range of averaging Hall voltage. (b) Magnitude comparison of three spin transistors. We compare a conventional Datta-Das spin-FET (two ferromagnets (FMs)), a spin Hall transistor (one FM \& one Spin Hall structure), and a ferromagnetic-free spin transistor (two Spin Hall structures). All spin transistors are made of an identical channel structure. 

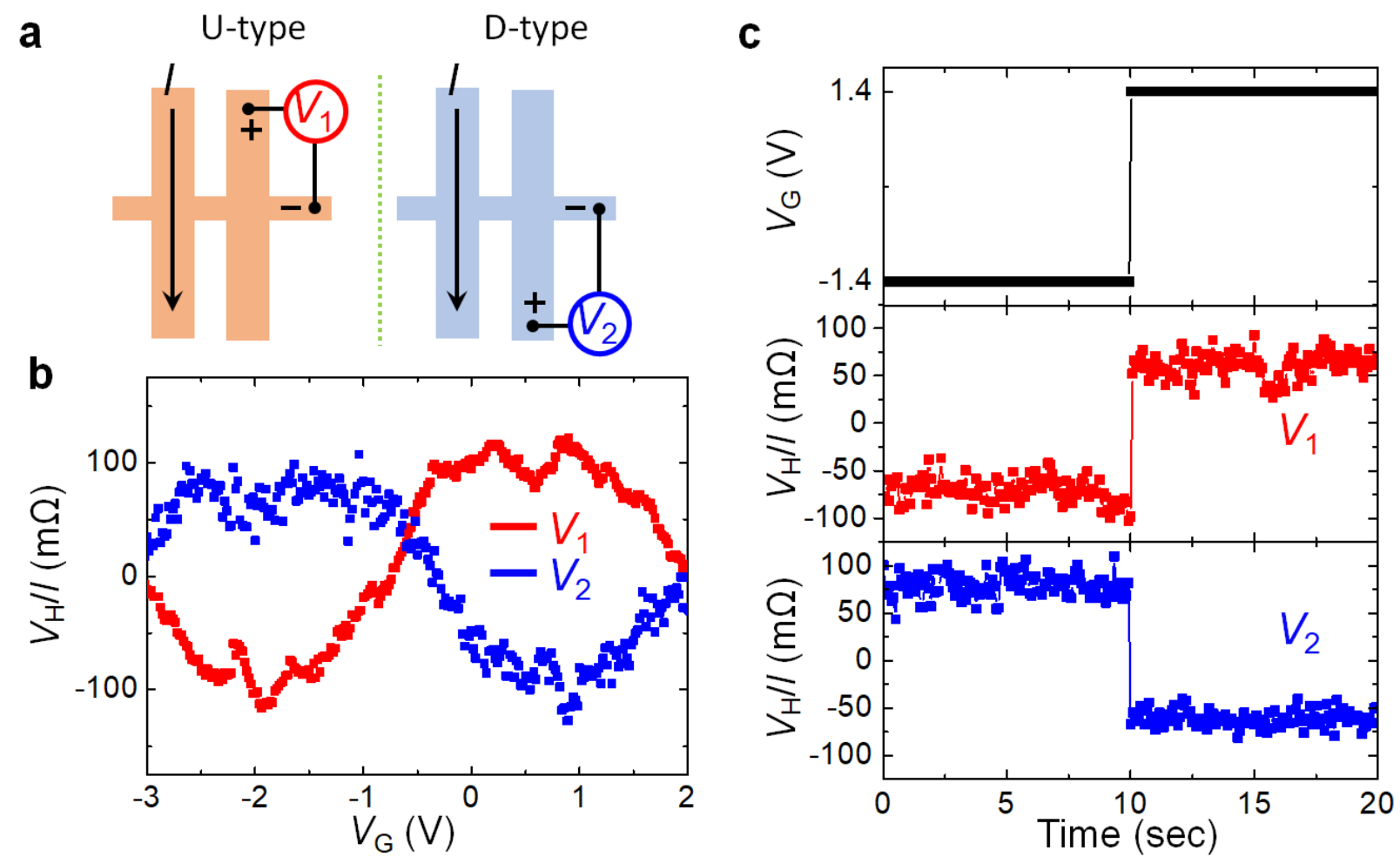

Figure 4. Complementary operation of ferromagnet-free spin Hall transistors. (a) Schematic structure of complementary transistors. (b) Gate-controlled signals of complementary transistors. (c) Example of logic application. The $U$ - and $D$-type spin transistors play roles of $n$ - and $p$-type conventional transistors. The channel length is $1.1 \mu \mathrm{m}$, bias current is $0.1 \mathrm{~mA}$, and the measuring temperature is $1.8 \mathrm{~K}$. The offset resistance is $0.8 \Omega$. 


\section{ASSOCIATED CONTENT}

\section{Supporting Information.}

The Supporting Information is available free of charge on the ACS Publications website at DOI:

Further information about materials and methods, spin transport simulation for various disorder strength and channel length, and spin Hall signal depending on probe width (PDF)

\section{AUTHOR INFORMATION}

\section{Corresponding Author}

*E-mail: hckoo@kist.re.kr

\section{Author Contributions}

H.C.K. planned the experiment and supervised the research. W.Y.C and H.C.K. fabricated the devices and collected the data. H.K., J.C. and S.H.H. contributed sample preparation. A.A., H.B.M.S, A.M. and K.-J.L. performed device simulation and gave theoretical background. All authors analyzed the data and wrote the manuscript.

\section{Notes}

The authors declare no competing interests.

\section{ACKNOWLEDGMENT}

This work was mainly supported by Samsung Research Funding Center of Samsung Electronics under project Number SRFC-MA1502-06. H.C.K acknowledge the KIST and KU-KIST 
Institutional Programs. A.M. and A.A. acknowledge support from the King Abdullah University of Science and Technology (KAUST). K.-J.L. acknowledges the KIST Institutional Program.

\section{REFERENCES}

1. Datta, S.; Das, B. Electronic analog of the electro-optic modulator. Appl. Phys. Lett. 1990, 56, 665-667. DOI: 10.1063/1.102730

2. Frolov, S. M.; Lüscher, S.; Yu, W.; Ren, Y.; Folk, J. A.; Wegscheider, W. Ballistic spin resonance. Nature 2009, 458, 868-871. DOI: 10.1038 /nature07873

3. Koo, H. C.; Kwon, J. H.; Eom, J.; Chang, J.; Han, S. H.; Johnson, M. Control of spin precession in a spin-injected field effect transistor. Science 2009, 325, 1515-1518. DOI: $10.1126 /$ science.1173667

4. Kohda, M.; Lechner, V.; Kunihashi, Y.; Dollinger, T.; Olbrich, P.; Schonhuber, C.; Caspers, I.; Bel'kov, V. V.; Golub, L. E.; Weiss, D.; Richter, K.; Nitta, J.; Ganichev, S. D. Gate-controlled persistent spin helix state in (In,Ga)As quantum wells. Phys. Rev. B 2012, 86, 081306(R). $\quad$ DOI: 10.1103/PhysRevB.86.081306

5. Sasaki, A.; Nonaka, S.; Kunihashi, Y.; Kohda, M.; Bauernfeind, T.; Dollinger, T.; Richter, K.; Nitta, J. Direct determination of spin-orbit interaction coefficients and realization of the persistent spin helix symmetry. Nature Nanotech. 2014, 9, 703-709. DOI: 10.1038/nnano.2014.128 
6. Manchon, A.; Koo, H. C.; Nitta, J.; Frolov, S. M.; Duine, R. A. New perspectives for Rashba spin-orbit coupling. Nature Mater. 2015, 14, 871-882. $\quad$ DOI: $10.1038 /$ nmat4360

7. D’yakonov, M. I.; Perel, V. I. Possibility of orienting electron spins with current. JETP Lett. 1971, 13, 467. DOI:

8. Kato, Y. K.; Myers, R. C.; Gossard, A. C.; Awschalom, D. D. Observation of the spin Hall effect in semiconductors. Science 2004, 306, 1910-1913. DOI: 10.1126/science. 1105514

9. Wunderlich, J.; Kaestner, B.; Sinova, J.; Jungwirth, T. Experimental observation of the spin-Hall effect in a two-dimensional spin-orbit coupled semiconductor system. Phys. Rev. Lett. 2005, 94, $047204 . \quad$ DOI: 10.1103/PhysRevLett.94.047204

10. Sih, V.; Myers, R. C.; Kato, Y. K.; Lau, W. H.; Gossard, A. C.; Awschalom, D. D. Spatial imaging of the spin Hall effect and current-induced polarization in two-dimensional electron gases. Nature Phys. 2005, 1, 31-35. DOI: 10.1038/nphys009

11. Saitoh, E.; Ueda, M.; Miyajima, H.; Tatara, G. Conversion of spin current into charge current at room temperature: Inverse spin-Hall effect. Appl. Phys. Lett. 2006, 88, 182509. DOI: $10.1063 / 1.2199473$

12. Garlid, E. S.; Hu, Q. O.; Chan, M. K.; Palmstrøm, C. J.; Crowell, P. A. Electrical Measurement of the Direct Spin Hall Effect in $\mathrm{Fe} / \mathrm{In}_{x} \mathrm{Ga}_{1-x}$ As Heterostructures. Phys. Rev. Lett. 2010, 105, 156602. DOI: 10.1103/PhysRevLett.105.156602 
13. Seki, T.; Hasegawa, Y.; Mitani, S.; Takahashi, S.; Imamura, H.; Maekawa, S.; Nitta, J.; Takanashi, K. Giant spin Hall effect in perpendicularly spin-polarized FePt/Au devices. Nature Mater. 2008, 7, 125-129. DOI: 10.1038/nmat2098

14. Fan, Y.; Upadhyaya, P.; Kou, X.; Lang, M.; Takei, S.; Wang, Z.; Tang, J.; He, L.; Chang, L.-T.; Montazeri, M.; Yu, G.; Jiang, W.; Nie, T.; Schwartz, R. N.; Tserkovnyak, Y.; Wang, K. L. Magnetization switching through giant spin-orbit torque in a magnetically doped topological insulator heterostructure. Nature Mater. 2014, 13, 699-704. DOI: $10.1038 /$ nmat3973

15. Mellnik, A. R.; Lee, J. S.; Richardella, A.; Grab, J. L.; Mintun, P. J.; Fischer, M. H.; Vaezi, A.; Manchon, A.; Kim, E.-A.; Samarth, N.; Ralph, D. C. Spin-transfer torque generated by a topological insulator. Nature 2014, 511, 449-451. DOI: 10.1038/nature13534

16. Chuang, P.; Ho, S.-C.; Smith, L. W.; Sfigakis, F.; Pepper, M.; Chen, C.-H.; Fan, J.-C.; Griffiths, J. P.; Farrer, I.; Beere, H. E.; Jones, G. A. C.; Ritchie, D. A.; Chen, T.-M. Allelectric all-semiconductor spin field-effect transistors. Nature Nanotech. 2015, 10, 35-39. DOI: $10.1038 /$ nnano.2014.296

17. Fert, A.; Jaffres, H. Conditions for efficient spin injection from a ferromagnetic metal into a semiconductor. Phys. Rev. B 2001, 64, 184420. DOI: 10.1103/PhysRevB.64.184420

18. Kamalakar M. V.; Dankert A.; Kelly P. J.; Dash S. P. Inversion of Spin Signal and Spin Filtering in FerromagnetlHexagonal Boron Nitride-Graphene van der Waals Heterostructures. Sci.Rep. 2016, 6, 21168 DOI: 10.1038/srep21168 
19. Dankert A.; Dash S. P. Electrical gate control of spin current in van der Waals heterostructures at room temperature. Nature Commun. 2017, 8, 16093 DOI: $10.1038 /$ ncomms 16093

20. Zhao, H.; Loren, E. J.; Van Driel, H. M.; Smirl, A. L. Coherence control of Hall charge and spin currents. Phys. Rev. Lett. 2006, 96, 246601. $\quad$ DOI: 10.1103/PhysRevLett.96.246601

21. Wunderlich, J.; Park, B.-G.; Irvine, A. C.; Zârbo, L. P.; Rozkotová, E.; Nemec, P.; Novák, V.; Sinova, J.; Jungwirth, T. Spin Hall effect transistor. Science 2010, 330, 1801-1804. DOI: $10.1126 /$ science.1195816

22. Valenzuela, S. O.; Tinkham, M. Direct electronic measurement of the spin Hall effect. Nature 2006, 442, 176 DOI: $10.1038 /$ nature04937

23. Choi, W. Y.; Kim, H.-j.; Chang, J.; Han, S. H.; Koo, H. C.; Johnson, M. Electrical detection of coherent spin precession using the ballistic intrinsic spin Hall effect. Nature Nanotech. 2015, 10, 666-670. DOI: 10.1038/nnano.2015.107

24. Jacquod, P.; Whitney, R. S.; Meair, J.; Büttiker, M. Onsager relations in coupled electric, thermoelectric, and spin transport: The tenfold way. Phys. Rev. B 2012, 86, 155118. DOI: $10.1103 /$ PhysRevB.86.155118

25. Nitta, J.; Akazaki, T.; Takayanagi, H.; Enoki, T. Gate control of spin-orbit interaction in an inverted $\operatorname{In}_{053} \mathrm{Ga}_{047} \mathrm{As} / \mathrm{In}_{0.52} \mathrm{Al}_{048} \mathrm{As}$ heterostructure. Phys. Rev. Lett. 1997, 78, 1335. DOI: 10.1103/PhysRevLett.78.1335 
26. Lo, I.; Tsai, J. K.; Yao, W. J.; Ho, P. C.; Tu, L.-W.; Chang, T. C.; Elhamri, S.; Mitchel, W. C.; Hsieh, K. Y.; Huang, J. H.; Huang, H. L.; Tsai, W.-C. Spin splitting in modulationdoped $\mathrm{Al}_{x} \mathrm{Ga}_{1-x} \mathrm{~N} / \mathrm{GaN}$ heterostructures. Phys. Rev. B 2002, 65, 161306(R). DOI:

10.1103/PhysRevB.65.161306

27. Hu, C.-M.; Nitta, J.; Jensen, A.; Hansen, J. B.; Takayanagi, H. Spin-polarized transport in a two-dimensional electron gas with interdigital-ferromagnetic contacts. Phys. Rev. B 2001, 63, 125333. DOI: 10.1103/PhysRevB.63.125333

28. Sunko, V.; Rosner, H.; Kushwaha, P.; Khim, S.; Mazzola, F.; Bawden, L.; Clark, O. J.; Riley, J. M.; Kasinathan, D.; Haverkort, M. W.; Kim, T. K.; Hoesch, M.; Fujii, J.; Vobornik, I.; Mackenzie, A. P.; King, P. D. C. Maximal Rashba-like spin splitting via kinetic-energy-coupled inversion-symmetry breaking. Nature 2017, 549, 492. $\quad$ DOI: $10.1038 /$ nature 23898

29. Zhang, S.-j.; Ji, W.-X.; Zhang, C-w.; Li, P.; Wang, P.-j. Two-dimensional large gap topological insulators with tunable Rashba spin-orbit coupling in group-IV films. Sci. Rep. 2017, 7, 45923. DOI: 10.1038/srep45923 